\title{
Stem Cell Research: Medical Applications, Ethical and Legal Controversies
}

\author{
Neelam Saba ${ }^{1}$ and Wahied Khawar Balwan ${ }^{2 *}$
}

${ }^{1}$ Assistant Professor, Department of Zoology, Govt. Degree College Doda, Jammu and Kashmir, India

${ }^{2}$ Assistant Professor, Department of Zoology, Govt. Postgraduate College Bhaderwah, Jammu and Kashmir, India

DOI: $10.36348 /$ sjbr.2021.v06i02.006

| Received: 12.02.2021 | Accepted: 21.02.2021 | Published: 26.02.2021

*Corresponding author: Dr. Wahied Khawar Balwan

\section{Abstract}

Stem cells are special kind of cell from an embryo, foetus or adult, capable of renewing itself under certain conditions and of becoming specialised cells that make up the different tissues and organs of the body. They may be isolated from embryos, umbilical cords and adult tissues. Stem cells from embryos can treat many diseases but they are extremely dangerous and unpredictable. However, this power and versatility of these cells to produce different cell types has medical applications in cell therapies. Embryonic stem cell are harvested but this requires the destruction of human embryos, which is considered immoral and unethical, thus becoming contentious and giving rise to ethical controversies. However, stem cells isolated from adult tissues and created from skin cells addresses these issues. Most recently, they have been produced by reprogramming skin cells, an advance revolutionizing this field. Stem cell therapies have great potential. Stem cells provide an ideal model for studying the development of an organism and have the ability to replace damaged cells in the body that would otherwise not be replenished.

Keywords: Stem Cell, Foetus, Umbilical Cord, Embryo, Cell therapies, Skin cells.

Copyright () 2021 The Author(s): This is an open-access article distributed under the terms of the Creative Commons Attribution 4.0 International License (CC BY-NC 4.0) which permits unrestricted use, distribution, and reproduction in any medium for non-commercial use provided the original author and source are credited.

\section{INTRODUCTION}

Until quite recently, it was believed that the human body could not regenerate neurons- we could grow new skin and blood cells to replace those we lost, but nerve cells that died were gone forever. Then, 20 years ago, embryonic stem cells were discovered in mice. In 1998, stem cells were isolated from a human embryo. Today many hope that these so-called universal cells (stem cells) hold the key to treating perhaps and even curing neurological disorders and many other diseases as well. Stem cells are special kind of cell from an embryo, foetus or adult, capable of renewing itself under certain conditions and of becoming specialised cells that make up the different tissues and organs of the body [1]. In dictionary 'stem' means the main ascending (going up) stalk of a plant, similarly, there are main cells that grow through time, a main stem from which other stem can branch out from [1]. Stem cells are special cells that have the ability to divide for an indefinite period and can give rise to a wide variety of specialized cell types [2]. This ability, known as developmental plasticity, is a common feature of fertilized eggs and early embryonic cells (blastomeres). A fertilized egg is totipotent, has the highest degree of developmental plasticity and can give rise to all of the body cells. Blastomeres have decreased level of plasticity and can only give rise to all body cells. Blastomeres have decreased level of plasticity and can only give rise to a limited range of cell types and are therefore called Pluripotent. As development progresses, individual cells can give rise to only a few cell types before assuming the final form of a specialized cell that can only give rise to other cells of its kind, hence are called multipotent.

Stem cell does not have any tissue specific structures that allow it to perform specialized functions. A stem cell cannot work with its neighbors to pump blood through the body (like a heart muscle cell); it cannot carry molecules of oxygen through the blood stream (like red blood cell); and it cannot fire electrochemical signals to other cells that allow the body to move or speak (like a nerve cell). However, unspecialized stem cells can give rise to specialized cells, including heart muscle cells, blood cells or nerve cells. Stem cells may be isolated from embryos, umbilical cords (pluripotent) and adult tissues (multipotent). Stem cells grow and divide indefinitely when placed in culture. This power and versatility of these cells to produce different cell types has application in cell therapies viz. Parkinson's disease, Alzheimer's disease, cardiovascular disease, spinal cord disorders and some cancer. Leukemia, a cancer 
affecting white blood cells, can be treated by replacing the cancerous cells with stem cells programmed to differentiate into healthy white blood cells $[1,2]$.

Stem cells can give rise to unspecialized cells. When unspecialized cells give rise to specialized cells, the process is called differentiation. Scientists are just beginning to understand the signals inside and outside cells that trigger stem cell differentiation. The internal signals are controlled by a cell's genes, which are interspersed across long strands of DNA, and carry coded instructions for all the structures and functions of a cell. The external signals for cell differentiation include chemicals secreted by other cells, physical contact with neighboring cells and certain molecules in the microenvironment.

All cells in the animal body possess the powers of reproduction and differentiation but some lose the ability to divide soon after birth. The brain, spinal cord, muscles, heart and kidney possess this developmental pattern of active cell division during embryogenesis with loss of this ability in this ability in the adult to ensure a particular size and shape. Damage, if caused, may lead to loss of life or else a transplant has to be done to save life. However, skin, liver and bone marrow retain the power of division throughout the life span of the individual. Red blood cells with a life span of about 120 days are worn out regularly but the stem cells in the bone marrow divide and differentiate both into red blood cells and white blood cells to replace them.

This concept that the stem cells in the bone marrow replace the worn out RBCs is being applied to repair other organs such as brain and heart which are incapable of repairing themselves. Stem cells from embryos can treat many diseases but they are extremely dangerous and unpredictable. This is possible only when embryonic stem cells are harvested but this requires the destruction of human embryos, which is considered immoral and unethical, thus becoming contentious and giving rise to ethical controversies. However, stem cells isolated from adult tissues and created from skin cells addresses these issues. Most recently, they have been produced by reprogramming skin cells, an advance revolutionizing this field. Stem cells therapies have great potential.

Stem cell research is focused on embryonic stem cells, adult stem cells, therapeutic cloning and induced pluripotent stem cells. The ultimate stem cell is the fertilized egg which can give rise to an entire organism consisting of hundreds of different kinds of cells. Human, mouse and amphibian blastomeres from two or four cell embryos also retain their totipotency and are good embryonic stem cells. Mammalian embryonic stem cells are obtained exclusively from the inner cell mass of a blastocyst (with or without killing the embryo), and when placed in cell culture they can differentiate into many kinds of cells representing all three embryonic germ layers. However, once the association between the inner cell mass and the trophoblast is disrupted, the embryonic stem cells cannot develop into an embryo [3].

Embryonic germ cells can also be collected from the gonadal ridge (located in the fetuses' lower mid-back) of 9-12 week old fetuses (killed during abortion). Cells in this area form the gonads of the adult which produce the germ cells (eggs or sperms). However, these cells and hence are less desirable in stem cell therapies.

Adult stem cells are collected from adult tissues or organs. The first Adult stem cells when cultured differentiate into a small range of cells representing one or two germ layers, in contrast to the Embryonic stem cells which can differentiated into a wide variety of cell types, representing all germ layers, however, they can be stimulated in vitro into a wide variety of cell types representing mesoderm, neuroectoderm and endoderm, the three fundamental germ layers. The Adult stem cells, hence, can also be called multipotent adult progenitor cells.

Blood isolated from human umbilical cords is an excellent source of Adult stem cells. Moreover, these umbilical cord stem cells have a developmental plasticity equal to that of embryonic stem cells. Umbilical cord blood banks are established by collecting blood from newborn infants in case the child should ever need stem cell therapy. The existence of Adult stem cells is extremely important since they resolve the ethical problems that are associated with the harvesting of Embryonic stem cells and it solves the problem of tissue rejection. Adult stem cells can be obtained from other organs, such as the pancreas, liver, which allow the production of a wider variety of cell types [1].

Therapeutic cloning involves cloning the patient, isolating cloning the patient, isolating stem cells from the resulting embryos and using those cells to treat the same patient. The nucleus from a skin cell is introduced into an enucleated egg cell (oocytes). This procedure known as somatic cell nuclear transfer depends on the ability of the skin cell nucleus to sustain normal development and the production of a healthy embryo. Ian Wilmut cloned a sheep named Dolly in mammary gland epithelial cell to an enucleated egg. The cloned stem cells and any cells they give rise to, would carry the patient's unique cell-surface glycocalyx and consequently would not be attacked by the immune system. It, however, involved killing of embryos, does not produce patient specific embryonic stem cells, is controversial and hence has bleak future $[1,2]$. 
Induced pluripotent stem cells are easy to produce patient-specific cells which do not require human eggs or the killing of human embryos. A skin cell was converted by Shinya Yamanaka into an Induced pluripotent stem equivalent to an Embryonic stem cell. He reprogrammed skin cells by infecting them with viruses carrying four gene specific transcription factors and these cells responded to directed differentiation, indicating the embryo-like character. Induced pluripotent stem cells with their infinite potential have the capacity to completely replace therapeutic cloning and human Embryonic Stem cell research [4].

\section{Medical Applications}

Stem cells can be used to treat and possibly cure a wide variety of diseases. Diseases are treated by introducing whole human cells into the body in order to restore the patient's heath. The exception is induced pluripotent stem cells, stem cells produced using gene therapy.

\section{Cardio Vascular Disease}

It is caused by a chronic reduction in the blood supply to the heart leading to a cardiac infarction or heart attack [5]. If the extent of damage is less, the heart could be weakened butt still functional. Conditions like dilated cardiomyopathy, coronary syndromes, arrhythmias, hypertensive heart failure are reversible [6]. However, serious obstruction of the coronary arteries leads to the death of the cardiac muscle. At present, the only treatment for the massive and normally irreversible cardiac failure, not leading to death, is an organ transplant. Scientists are trying to develop an alternative cell based therapy using embryonic stem cells, adult stem cells and induced pluripotent stem cells.

Autogenic (from the patient) adult stem cells isolated from their own bone marrow when injected into the heart of patients, develop a tissue consisting of some cardiomyocytes and vascular structures. However, most of the injected adult stem cells fail to differentiate into functional cardio myocytes but a very modest improvement of cardiac function is observed due to the secretion of growth factors and other substances from the adult stem cells. Moreover, the macrophages eat up the transplanted cells also as they try to clean up the damaged cardiomyocytes in the scar tissue after a heart attack [7]. Use of embryonic stem cells has ethical problems in addition to the problems of production of teratomas and the induction of graft-versus-hostdisease, since the embryonic stem cells would be allogenic (not from the patient), induced pluripotent stem cells usage also has problems as they must be induced to a partially differentiated state before being injected into the patient. More precise methods are needed for directed differentiation in order to produce very homogenous cell populations and also to screen, and remover undifferentiated cells.

\section{Diabetes}

Diabetes is a chronic metabolic disorder that destroys the body's ability to utilize glucose, the uptake of which is regulated by a hormone called insulin that is produced by the $\beta$-cells of pancreas. Patients with Type-1 diabetes (insulin-dependent diabetes) have complete lack of insulin, while patients with Type- 2 diabetes (insulin-resistant diabetes) have too little insulin or their bodies do not use insulin effectively which may result in too high levels of insulin of blood. In Type- 1 diabetes, $\beta$-cells lose the ability to manufacture and release insulin, leading to a buildup of glucose in the blood. A chronic elevation of blood glucose levels results in inappropriate glycosylation (addition of sugar to proteins) of many proteins in the blood, including haemoglobin. Diabetes is currently treated with daily injections of purified insulin but it does not cure the disease or remove the long-term threat of kidney failure or the other associated complication. Stem cells are being used to cure the disease by directing the differentiation of cultured embryonic stem cells into $\beta$-cells that secrete insulin. But unfortunately, teratomas develop and risk giving the patient cancer, which may not be treatable. Adult stem cells and induced pluripotent stem cells are also being tried in this regard with the aim to return them to the patient in the hope that they will colonize the pancreas, thus curing the disease [8].

\section{Immune Deficiencies}

The immune system combats invading microbes with its enormous population of white blood cells mostly in the form of B-cells, T-cells and macrophages. $\mathrm{B}$ and $\mathrm{T}$-cells are lymphocytes that develop in the bone marrow and thymus respectively. B-cells attack microbes indirectly by producing antibodies. T-cells control and coordinate the immune response by releasing cytokines that recruit macrophages and B-cells. Macrophages are phagocytic blood cells that eat the invading microbes. A combination of stem cells and gene therapy is being used to cure immune deficiencies. The bone marrow from affected patients is extracted and transferred with hematopoietic stem cells and then reimplanted into the patient with the aim of developing a functional immune system.

\section{Leukemia}

Leukemia is a cancer of blood cells. If affects white blood cells only and can arise in either lymphoid cells (lymphocytic leukemia) or myeloid cells (myelogenous leukemia). The disease has two forms namely acute and chronic. Acute leukemia progresses very quickly and usually destroys the patient's immune system. Chronic leukemia progresses much more slowly and even though the leukocytes are transforming, they retain some of their normal functions, so the immune system is not destroyed so quickly or so completely. The standard treatment involves radiation and chemotherapy which kill the 
cancerous cells. Extreme forms of this therapy involve the complete destruction of bone marrow with radiation therapy, after which the patient receives new bone marrow from a suitable donor, which is very difficult to get. Stem cell therapy is now used to treat all forms of leukemia with autologous transplants, thus removing the need to find bone marrow donors. Stem cells, isolated from bone marrow of the affected patient are induced to differentiate into white blood cell precursors and then grown in culture to increase their numbers. Once these cells are collected, the patient's cancerous bone marrow is destroyed and the stem cell derived blood cells are returned to the patient in order to reconstitute a healthy, cancer-free bone marrow [5].

\section{Liver Disease}

Liver converts ammonia to urea which passes out of our bodies are urine. The production of urea depends on the liver enzyme ornithine trans carbamylase. If this enzyme is defective, blood levels of ammonia increase rapidly, resulting in coma, brain damage and death. Hematopoietic stem cells isolated from the patient and stimulated to differentiate into liver cells can be reintroduced into the patient. These partially differentiated stem cells will colonize the liver and produce enough liver enzymes to cure the disease. These bone marrow derived stem cells can effectively rescue experimental liver failure and contribute to liver regeneration [5].

\begin{abstract}
Alzheimer's Disease
Alzheimer's Disease is a neurological disorder/neurodegenerative disease affecting the central nervous system which leads to a progressive loss of memory, language and the ability to recognize friends and family, it is often related to a biochemical or neurotransmitter defect believed to be caused by a combination of genetics, lifestyle and environment. With this disease the brain actually shrinks as more of the brain cells die off. The central nervous system is divided into the cerebrum, called the hippocampus is important for coordinating memory functions. This disease mostly begins in the hippocampus/cerebral cortex and also in certain subcortical regions [9]. During the early stages some damage occurs to the brain but not enough to produce outward signs of the disease. Over a period, it spreads to many areas of the cerebrum leading to the confusion and loss of memory that accompany the disease. Defects in the genes associated with the disease lead to the extensive death of neurons that is characteristic of this disease. Stem cells stimulated to differentiate into neurons and glia cells, and injected directly into the brain are thought to repair the damage to the brain.
\end{abstract}

\section{Parkinson's Disease}

Parkinson's Disease is a neurodegenerative disease affecting neurons in the area of the brain called the substantia nigra that results in tremors, muscular stiffness, and difficulty with balance and walking.
Dopaminergic neurons (neurons producing a neurotransmitter called dopamine) in the substantia nigra are damaged in this disease. Since the neurological damage caused by the Parkinson's disease is restricted to one region of the brain, stem cell therapy may be successful in treating this disease. Preclinical research has shown that it is possible to isolate stem cells that can be stimulated to differentiate into dopaminergic neurons. Injection of these neuronal stem cells into affected mice relieved some of the disease symptoms, particularly the loss of motor control that is characteristic of this disease. However, non-motor symptoms like olfactory dysfunctions, cognitive impairments, sleep dependently along with motor symptoms have also been reported $[1,10]$.

\section{Spinal Cord Injury}

Damage to the spinal cord caused by automobile accidents or falls from high places can make it impossible for the brain to control the extremities and internal organs such as heart and lungs. The severity of the damage depends on how close to the brain the spinal cord injury is. If the individual's neck is broken, he or she may end up being a quadriplegic (a person unable to move the arms and legs) and may not be able to breathe properly. If the damage to the spinal cord is near the middle of the neck the patient will be paraplegic (unable to move the legs) but will retain control over the arms and lungs. Stem cell therapy aims to repair the damage by providing neurons to reestablish the circuit and oligodendrocytes for insulation. Adult stem cells usage would make it possible to treat patients without the fear of immune rejection $[3,11]$.

\section{Stroke}

A stroke occurs when the blood supply to the brain is interrupted, leading to the death of brain cells. As a result, the affected part of the brain is unable to function, leading to partial or complete paralysis of various parts of the body. A stroke can lead to permanent neurological damage and is often fatal. Research has shown that it is possible to activate the body's own stem cells by injecting transforming factor $\alpha(\mathrm{TF}-\alpha)$, to repair neural damage caused by stroke.

\section{Ethical Controversies}

Stem cells offer the hope of curing disease and repairing a damaged nervous system. But the strategies require the destruction of human embryos and in its most controversial form (therapeutic cloning), the creation of human embryos for the sole purpose of providing stem cells. This gives rise to many ethical problems in biomedical research.

\section{Justice and Beneficence}

Beneficence refers to acts of kindness or charity. In biomedical research, this means never injuring one person to benefit another, justice should ensure that researches should never enlist subjects in an experiment if those subjects do not stand to reap any 
benefits. Researches can enroll human subjects in a clinical research trial only if the procedure is carefully explained and the researchers receive written consent from the prospective subjects. A patient advocate also needs to be present in this process to ensure that the full disclosure of background research is provided, results that may suggest the subjects will be armed by the experiment informed and finally ensure that there is no attempt to coerce prospective subjects into joining the trial.

\section{High-Tech Cannibalism}

Opponents of embryonic stem cell research, particularly in the form of therapeutic cloning, believe it is wrong to use human embryos for any kind of research or medical therapy and have called this as high-tech cannibalism. The issue of embryonic stem cell research is extremely complex and involves the very emotional and highly politicized issue of human abortion. Researchers want unlimited access to human embryos left over from in vitro fertilizations to use as a source of stem cells. Scientists want to use therapeutic cloning to create human embryos using nuclear transfer for the sole purpose of providing stem cells and ultimately culture grown kidneys and other organs. Embryos do not survive the harvesting of the stem cells; are embryos.

Critics argue that since neither the embryo nor the foetus is recognized as a person, in the legal sense, there is no obligation for embryonic stem cell researchers to abide by any research policies. However, the absence of legal status does not preclude an ethical policy, as evidenced by the fact that abortion is only permissible up to the fifth month and not beyond (except in very rare cases where the mothers' life is at risk), even though the foetus does not attain the legal status of a person until after birth. With this in mind opponents of therapeutic cloning and embryonic stem cell research maintain that at the very least, a human embryo or foetus, incapable of giving informed consent, should be afforded the benefit of an advocate. Resistance to this notion centres on the question of when an embryo or a foetus becomes human [3].

\section{On Becoming Human}

The question of when an embryo becomes human is still debated. Public perception and dialogues ever since believe that an embryo did not become human until after birth. Science ethicists state that cloned human embryos, destined for state that cloned human embryos, destined for therapeutic research, are not really human. Some scientists, following the logic of genomes, state that an embryo is human from the day of conception. The in vitro fertilization embryos are produced for the purpose of creating life i.e. they are all intended for implantation and subsequent development and hence can be allowed with tight regulations. Therapeutic cloning creates embryos for the sole purpose of harvesting stem cells, thus killing the embryo in the process and hence it is inherently unethical and should be banned.

\section{Legal Controversies}

Embryonic stem cell research may provide powerful new methods for treating a variety of medical disorders. However, it also introduces many ethical problems that require legislation to control its use and spread. The legal issues deal with stem cells that are harvested from three-to-five day-old human embryos, donated by IVF clinics, or produced by therapeutic cloning. There are no legal issues associated with the use of adult stem cells, induced pluripotent stem cells, or stem cells isolated from umbilical cord blood. Therapeutic cloning represents a fusion of cloning technology and stem cell research and its regulation is an extremely different problem.

The regulation of stem cell research in the United Kingdom is governed by the Human Fertilization and Embryology Act of 1990. Under this act, research on embryos older than 14 days is prohibited. This time period was set to coincide with the appearance of the primitive streak, an anatomical feature of an embryo that indicates the beginning of neurulation and the formation of the central nervous system. Research on non-human embryos or by other means is preferred. Research is allowed only for promoting advances in the treatment of infertility, increasing knowledge about the causes of congenital diseases and miscarriages, creation and development of embryos, development of contraceptives, for developing methods for detecting the presence of gene or chromosome abnormalities in embryos before implantation and in developing medical therapies. However, reproductive cloning, whereby an embryo is produced by nuclear transfer and then carried to term by a surrogate mother is banned.

In the United States, human stem cells research is funded only for the ES cells isolated from embryos created at IVF clinics for reproductive purposes with the consent of the do not. Funding is not provided for embryonic stem cells isolated from other sources such as therapeutic cloning, IVF embryos created specifically for research purpose, development of an unfertilized egg, and human-animal hybrid embryos [3].

\section{CONCLUSION}

Studying stem cells has great social issues associated with it. Scientists are interested in using stem cells to grow whole organs in the laboratory that could be used to replace defective organs. It is feasible to do this and it will go a long way towards relieving the chronic shortage of organ supplies for transplant surgery. However, it is extremely difficult and would take more than 20 years to produce a functional organ. Stem cell therapy holds the potential for curing terrible diseases. It offers hope to those paralyzed by a spinal 
cord injury and someday it may be used to reverse the ageing process.

Embryonic stem cells are isolated from two-tofive day old human embryos which do not survive the harvesting of the stem cells, and many people believe that it is highly immoral to kill a human embryo for its cells. Scientists prefer embryonic stem cells for medical therapies because they possess a high degree of developmental plasticity and when injected into experimental animals the damage is repaired best. However, it is difficult to control the growth of these cells and these may form cancerous tumors called teratomas. Immune rejection is a very serious problem with embryonic stem cell therapy, since these cells are not related to the patient. Adult stem cells are isolated from adult tissues such as bone marrow and from umbilical cord blood. Induced pluripotent stem cells are produced in the laboratory by reprogramming skin cells taken from the patient needing treatment, and thus there is no threat of immune rejection with these cells or with adult stem cells. The use of embryonic stem cells is very controversial, whereas the use of adult stem or induced pluripotent stem cells is not.

\section{REFERENCES}

1. Jain, Y.C. (2008). Stem Cells. Biology spectrum, January, 61-63.

2. Kishore, P.V.S. (2017). Stem Cell Research. Everyman's Science, LII (1): 46-52.

3. Joseph, P. (2010). Stem cell Research, InfoBase Publishing, New York, USA.
4. Takahashi, K., \& Yamanaka, S. (2006). Induction of pluripotent stem cells from mouse embryonic and adult fibroblast cultures by defined factors. Cell, 126(4):663-676.

5. Orlic, D., Kajstura, J., Chimenti, S., Jakoniuk, I., Anderson, S.M., Li, B., Pickel, J., McKay R., Nadal-Ginard, B., Bodine, D.M., Leri, A., \& Anversa, P. (2001). Bone marrow cell regenerate infarcted myocardium. Nature, 410 (6829): 701705.

6. Bhupatiraju, S.N., \& Tucker, K.L. (2011). Stem Cell Research. Clinica Chimica Acta, 412, (17-18): 1493-1514.

7. Segers, F.M.V., \& Lee, R.T. (2008). Stem cell therapy for cardiac disease. Nature, 451: 937-942.

8. Surani, M.A., \& McLaren, A. (2006). Stem Cells: a new route to rejuvenation, Nature, 443(7109): 284285.

9. Cheng, S.T. (2016). Cognitive Reserve and the prevention of Dementia: the role of physical and cognitive activities. Current Psychiatry Reports, 18(9): 85.

10. Petrelli, A., Kaesberg, S., Barbe, M.T., Timmermann, L., Fink, G,R., Kessler, J., \& Kalbe, E. (2014). Effects of cognitive training in Parkinson's disease: a randomized controlled trial. Parkinsonism and Related Disorders, 20(11):11961202.

11. Wadman, M. (2009). Stem Cells ready for prime time. Nature, 457: 516.

12. Guyton., \& Hall. (2012). Textbook of Medical Physiology, 950-951, Saunders Elsevier, Philadelphia. 